\title{
卟啉敏化太阳能电池研究进展
}

\author{
顾承志 ${ }^{a, b}$ 孟舒献 ${ }^{a}$ 冯亚青*,a,c \\ ( ${ }^{a}$ 天津大学化工学院 天津 300072 ) \\ ( ${ }^{b}$ 石河子大学化学化工学院 石河子 832000) \\ $\left({ }^{c}\right.$ 天津化学与化工协同创新中心 天津 300072$)$
}

\begin{abstract}
摘要 由于自然界中光合作用的核心结构是卟啉, 其具有良好的光、热和化学稳定性, 在过去的二十年中卟啉染料敏 化太阳能电池研究获得了极大的关注, 2014 年卟啉染料光电转换效率突破了 $13 \%$, 成为新的基准效率. 以光电性能为 主线, 从分子工程设计的角度出发, 系统性地总结了应用于敏化太阳能电池的不同结构卟啉染料性能的研究进展. 关键词＼cjkstart染料敏化太阳能电池; 卟啉; 分子工程设计; 光电性能
\end{abstract}

\section{Progress of Porphyrin Sensitizers for Dye-Sensitized Solar Cells}

\author{
Gu, Chengzhi ${ }^{a, b} \quad$ Meng, Shuxian ${ }^{a} \quad$ Feng, Yaqing*,a,c \\ ( ${ }^{a}$ School of Chemical Engineering, Tianjin University, Tianjin 300072) \\ ( ${ }^{b}$ School of Chemistry and Chemical Engineering, Shihezi University, Shihezi 832000) \\ ( ${ }^{c}$ Collaborative Innovation Center of Chemical Science and Engineering, Tianjin 300072)
}

\begin{abstract}
As a core structure of nature photosynthesis, porphyrin has good light, thermal and chemical stability. In the past two decades, porphyin based dye-sensitized solar cells (DSSC) has attracted much attention relevant to global environmental issues. Up to date, they exhibit the best performance with a benchmark efficiency of $13 \%$ reported in 2014 for dye-sensitised solar cells. The progress of porphyrins and their derivatives applied in DSSC from the point of view of molecular engineering design correlated with photovoltaic performance is systematically summarized.

Keywords dye-sensitized solar cells; porphyin; molecular engineering design; photovoltaic performance
\end{abstract}

化石能源的不可持续性，并且由化石能源过度消耗 所导致的环境持续性恶化, 使得开发低价、高效太阳能 光伏技术成为全球广泛关注的焦点 ${ }^{[1,2]}$. 相较传统的结 构钢性、制造工艺复杂、上游制造环境危害巨大的硅太 阳能电池, 由瑞士联邦理工学院 Grätzel 教授建立的染 料敏化纳米晶太阳能电池-料敏化太阳能电池(DSSC) (又称 Grätzel 电池)成为最有希望的光伏技术之一 ${ }^{[3,4]}$.

自 1991 年 Grätzel 研究组 ${ }^{[5]}$ 在 Nature 上报道的首个 三核联吡啶钓染料, 短短两年后, Grätzel 研究组 ${ }^{[6]}$ 报道 了一个光电转换效率达到了 $11.03 \%$ 染料, 俗称 “红染 料”N3. 以后的十年间, 主要是 Grätzel 研究组为使联吡 啶钓染料在太阳光谱中有更宽的吸收以及解决热稳定 问题, 在 N3 的基础上进一步修饰, 几个光电转换效率
接近或超过 $10 \%$ 著名染料相继问世，他们是 N749: Black $\mathrm{dye}^{[7]}$ (光电转换效率 $\eta=11.1 \%, 2001$ 年)、 $Z 907^{[8 \sim 11]}\left(\eta=9.5 \%, 2002\right.$ 年) $\mathrm{N} 719^{[12]}(\eta=11.18 \%, 2005$ 年)以及 $\mathrm{C} 106^{[13,14]}(\eta=11.7 \%, 2010$ 年). 随着这几个染料 在染料敏化太阳能电池研究中广泛应用, 人们对染料敏 化纳米晶半导体太阳能电池的工作原理也有了较深的 理解. 追逐上述著名的 5 个联吡啶钓配体染料的结构修 饰思路，众多结构多样的染料被合成并进行光电性能研 究, 其目的是寻求高摩尔消光系数和高稳定性的染料敏 化剂. 研究思路被借鉴到其它类型的不含金属的有机敏 化染料中, 形成了二十年来染料敏化纳米晶 $\mathrm{TiO}_{2}$ 太阳 能电池研究热潮. 报道 DSSC 文献超过 1000 篇以上, 但 是光电转换效率停滞不前, 除了联吡啶钓配体染料, 其

\footnotetext{
*E-mail: yqfeng@tju.edu.cn
}

Received December 11, 2014 ; revised January 5, 2015 ; published online February 2, 2015.

Project supported by the National Natural Science Foundation of China (Nos. 21076147, 21476162) and the National International S\&T Cooperation Foundation of China (No. 2012DFG41980).

国家自然科学基金(Nos. 21076147, 21476162)、国家国际科技合作专项(No. 2012DFG41980)资助项目. 
它的有机染料的光电转换效率鲜有超过 $10 \%$, 以致于英 国巴斯大学的 Peter 等 ${ }^{[15]}$ 提出这样尖锐的问题 “The Grätzel Cell: Where Next?” 。虽然基于联吡啶钌 DSSC 在理论上以及实验室应用上有极大进展，但钉金属稀 有、价高并且可能泄漏引起一系列的环境问题, 以及联 吡啶钌染料的规模制备问题，限制了其实践应用，人们 不得不寻求更安全、更经济的光敏染料 ${ }^{[16 a, 16 b]}$.

地球上直接利用太阳能进行光电化学能转换的生 物途径就是光合作用, 而这些生命体中光反应中心的核 心结构都含有金属镁配位的卟吩核结构(图 1).

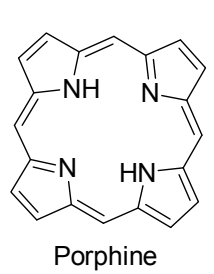

卟吩

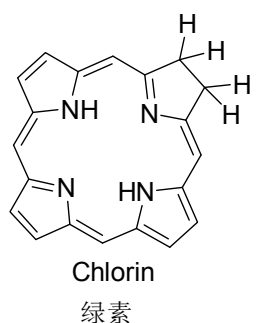

绿素

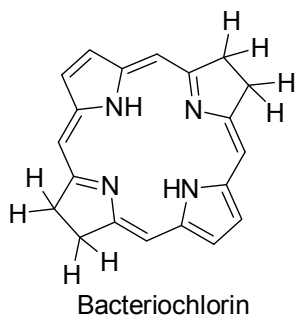

菌绿素
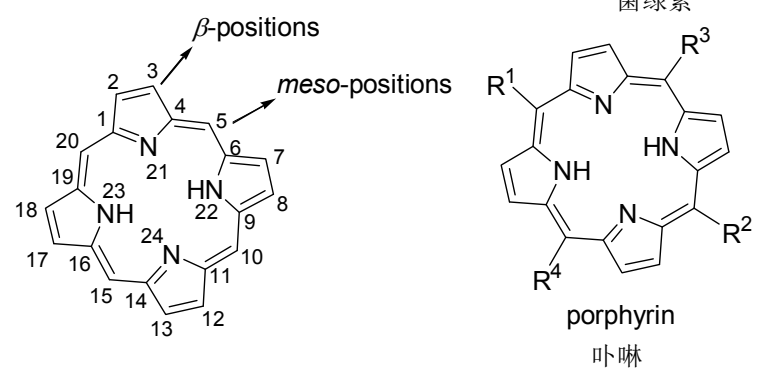

图 1 卟吩、类卟吩和卟啉结构

Figure 1 Structures of porphine, porphine-type and porphyrin

卟吩核结构的 $\beta$ 位和中位(meso)被其它的基团修饰 后就构成了卟啉化合物, 因此卟啉是一类由 4 个吡咯环 通过次甲基相连形成的具有大 $\pi$ 电子的芳香共轭大环化 合物, 是一类非常重要的电子给体, 卟啉自身由于大的 环电流，因而导电性能很差，一旦和金属配位后就成了 有机半导体, 构成其具有光导性的基本条件. 其中心的 氮原子与金属原子配位形成 $1: 1$ 金属卟啉配合物. 卟 啉化合物及其金属配合物常常在可见光区 $400 \sim 450 \mathrm{~nm}$ 附近有强烈的光谱响应以及 $550 \sim 650 \mathrm{~nm}$ 附近有中等强 度的吸收, 具有良好的光、热和化学稳定性; 卟啉结构 的 8 个 $\beta$ 位和 4 个中位 (meso) 可供官能团化, 为精细调 控卟啉染料的光学、电化学、光伏性质提供了广阔的空

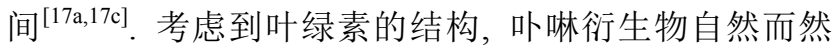
作为一类极具潜力、环境友好的光电转换材料成为国际 上人们研究的热门领域 ${ }^{[18 a, 18 b, 19,20 a, 20 b]}$.

\section{2 国内外研究现状}

1993 年, Grätzel 研究组 ${ }^{[21]}$ 首次报道了一个衍生于叶 绿素结构的铜卟啉 IX 染料(图 2), 其光电转换效率只有
$2.6 \%$, 而此时 $\mathrm{N} 3$ 染料的光电转换效率已达到 $11 \%$.

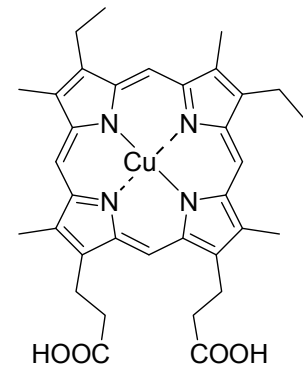

图 2 铜卟啉 IX 的结构

Figure 2 The structure of $\mathrm{Cu}$ mesoporphyrin IX

在随后的 10 年中卟啉染料进展缓慢，直到 2004 年， Officer, Grätzel 及合作者 ${ }^{[22]}$ 报道了一个锌卟啉染料，其 结构特点是, 卟啉的 $\beta$ 位通过反式乙烯基苯基与羧基相 连，其光电转换效率达到 $4.8 \%$, 一年后同一个组 ${ }^{[23]}$ 仅仅 在翔基的 $\alpha$-位增加一个㲵基，其光电转换效率提高到 5.8\%；2007 年他们 ${ }^{[24]}$ 继续对上述卟啉结构修饰，通过多 增加一个乙烯基以及把氧基替换成羧基得到的锌卟啉 染料, 其光电转换效率可达 $7.1 \%$. 如图 3 所示, 众所周 知联吡啶钉染料的吡啶环和叶绿素卟啉环上的的 $\beta$ 位都 有亲水性的极性基团羧基，并且羧基越多越好，后来很 多其它有机小分子或者聚合物的研究也表明了同样的 结果 ${ }^{[25]}$. 对于染料敏化太阳能电池，极性基团是非常必 要的，常常把这类基团称作 “针” 基团，其作用不仅仅 是针定在纳米晶二氧化钛上，更重要的意义在于扮演一 个电子受体, 否则就不可能发生电荷分离. 给电子基团 和 “针” 定基团之间通过刚性的大 $\pi$ 桥和桥接基团如乙 烯基、乙炔基、苯乙烯基(乙烯基苯基)、苯乙炔基(乙炔 基苯基)等相连，并且桥接基团的共轭键重复数目或者 说链长短都会对光电能量转换效率产生重大影 响 ${ }^{[26]}$. 锌卟啉分子的性能研究明确了从卟啉的 $\beta$ 位出发设计 D- $\pi$-A (Donor- $\pi$-Acceptor: 电子给体- $\pi$ 桥-电子受体)结 构体系为后来卟啉染料的设计奠定了基础.

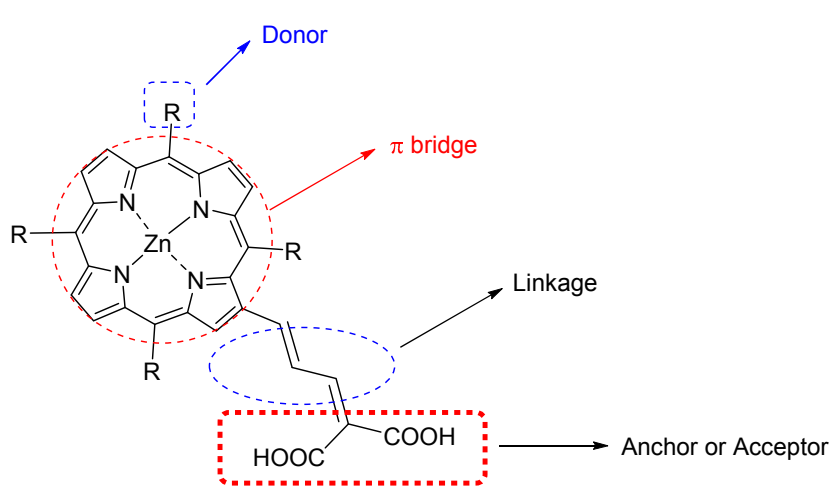

图 3 Grätzel 设计的桥接基团在卟啉 $\beta$ 位的染料结构

Figure 3 Porphyrin dye with linkers at $\beta$-positions by Grätzel group 
国内湘潭大学谭松庭研究组 ${ }^{[27]}$ 把噻吩基团引入 到桥接基团, 获得一个光电转换效率达 $5.14 \%$ 的染 料. 张宪胥、 Nazeeruddin 和 Grätzel 等 ${ }^{[28]}$ 合作, 用密度 泛函理论(DFT) 计算了 D- $\pi-A$ 体系中卟吩核上连有 $\mathrm{NH}_{2} 、 \mathrm{OH} 、 \mathrm{CH}_{3} 、 \mathrm{~F} 、 \mathrm{SH}$ 等取代基相应染料分子的基态 能量及光谱性质, 计算结果表明: $\mathrm{NH}_{2} 、 \mathrm{OH} 、 \mathrm{CH}_{3}$ 等取 代基能明显降低分子的 HOMO-LUMO 带宽, 显著增强 吸收红移, 以及好的前线轨道空间分布, 理论计算结果 为其后的卟啉结构修饰提供了新的思路.

经过几年的努力, 卟啉染料敏化太阳能电池取得了 一定的进展, 但是相对于联吡啶钉染料来说, 光电性能 还有较大的差距, 人们普遍认为吓啉染料自身的光吸收 范围窄问题可能是一个主要方面. 叶绿素由于卟啉的 meso 位与 $\beta$ 位存在并环的结构特点而有较宽的吸收. 从 2007 年起 Imahori 等 ${ }^{[29 ~ 32]}$ 陆续把卟啉 meso 位与 $\beta$ 位并 环结构修饰思路引入到卟啉敏化太阳能电池中(图 4), 虽然这些 $\pi$ 电子扩展卟啉染料吸收变宽了, 但是相应的 光电转换效率并没有显著提升, 一个原因, $\pi$ 电子扩展导 致卟啉聚集加剧, 从而抑制光子吸收, 另外卟啉染料分 子的尺寸增大降低了其在纳米 $\mathrm{TiO}_{2}$ 多孔膜上的吸附, 由于吸附染料分子的减小, 光子的吸收也相应减少了, 从而对其光电转换效率产生重大影响 ${ }^{[3,34]}$.

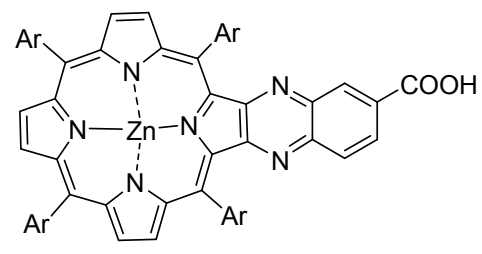

ZnQMA $\eta=5.2 \%$
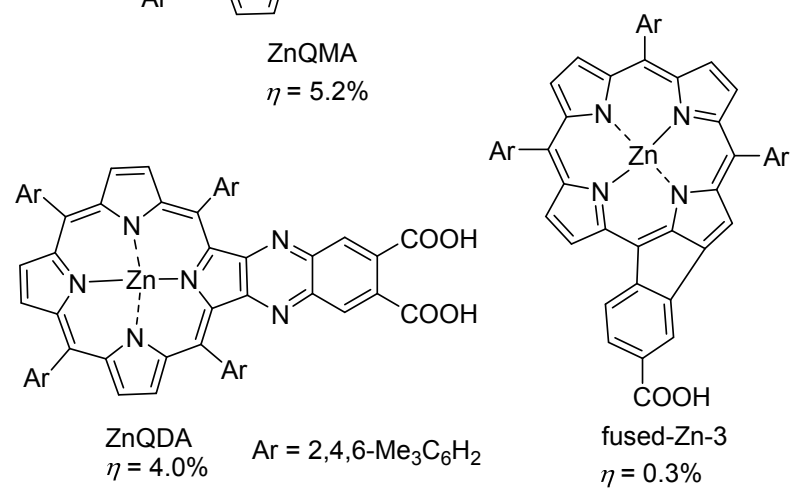

图 4 Imahori 使用卟啉的结构

Figure 4 The porphyrin dye by Imahori group

我们课题组 ${ }^{[35 \mathrm{a} \sim 35 \mathrm{e}]}$ 在研究吓啉方法学的同时也设计 了系列卟啉 $\eta$ 位稠合的染料(图 5)以及简单的 Trans-AB卟啉染料, 相应的光电性能证实了卟啉染料分子尺寸对 其效率的影响.

我们采取不对称卟啉经 1,3-偶极环加成反应设计合 成了 Chlorin 敏化染料 $\mathrm{ChD1a} \sim \mathrm{ChD}^{2} \mathrm{a}^{[36]}$ (图 6).

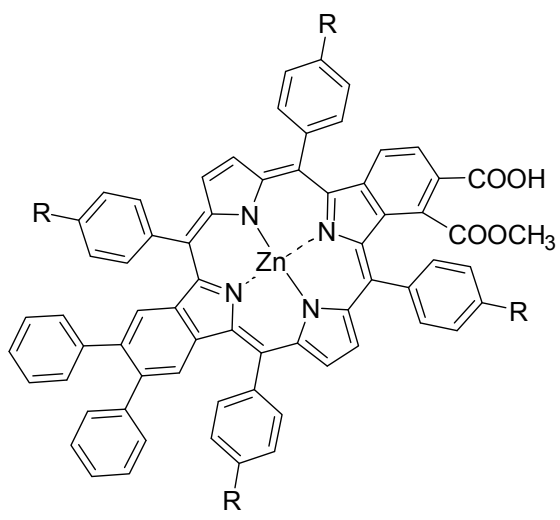

图 5 Feng 设计的卟啉染料结构

Figure 5 The porphyrin dye by Feng group

对于环加成位阻基团较小的 Chlorin 敏化染料 $\mathrm{ChD} 1 \mathrm{a} \sim \mathrm{ChD} 4 \mathrm{a}$ ，位阻基团与 $\mathrm{COOH}$ 呈现垂直关系且膜 紫外中 $\mathrm{Q}$ 带吸收强度越强的染料光电性能越好. 光电转 化效率以及 IPCE 的顺序: $\mathrm{ChD} 1 \mathrm{a}>\mathrm{ChD} 4 \mathrm{a}>\mathrm{ChD} 2 \mathrm{a}>$ $\mathrm{ChD} 3 \mathrm{a}$ ，而对于取代基为 2,6-二氯苯基(较小基团)的四 个 Chlorin 型染料, 光电转化效率以及 IPCE 的顺序: $\mathrm{ChD} 2 \mathrm{~b}>\mathrm{ChD} 1 \mathrm{~b}>\mathrm{ChD} 4 \mathrm{~b}>\mathrm{ChD} 3 \mathrm{~b}$.

以上的事实说明, 从卟啉的 $\beta$ 位引出电子受体的结 构修饰思路没有获得理想的光电转换效率突破，在这些 工作的基础上，Yeh、Diau 等 ${ }^{[37 \sim 45]}$ 设计了一系列中位 (meso 位) 修饰的锌卟啉染料, 其中如图 7 所示的 YD2-O$\mathrm{C} 8$ 结构, 当用三(联吡啶)钴 ${ }^{(\mathrm{II} / \mathrm{III})}\left[\mathrm{Co}^{(\mathrm{II} / \mathrm{III})}\right.$ tris(bipyridyl) $]$ 体 系替换常用的 $\mathrm{I}^{-} / \mathrm{I}^{3-}$ 体系, 获得了 $J_{\mathrm{sc}}=17.3 \mathrm{~mA} \cdot \mathrm{cm}^{-2}\left(J_{\mathrm{sc}}\right.$ 为短路电流密度), $V_{\mathrm{oc}}=965 \mathrm{mV}$ ( $V_{\mathrm{oc}}$ 为开路电压), $F F=$ 0.71 ( $F F$ 为填充因子), 其 $\eta$ 值达到 $11.9 \%$. 再用这个染 料与 Y123 共敏化, 进一步提高光电转换效率至 $12.3 \%$, 这是 2011 年底 DSSC 最高纪录. 这类锌卟啉染料结构修 饰思路如下：卟啉的一个中位直接与二芳胺基团的 $\mathrm{N}$ 原 子相连，二芳胺基团可以看作一个强电子给体，从而赋 予整个卟啉染料有更好的光吸收能力，卟啉相对的另一 个中位通过乙炔基苯基共轭桥与针定基团羧酸相连, 乙 炔基苯基共轭桥的引入同样会使相应卟啉的吸收光谱 进一步红移和变宽，而且乙炔基为线性分子，可能影响 卟啉染料在 $\mathrm{TiO}_{2}$ 膜上的吸附空间取向, 更有利于激发 电子注入到 $\mathrm{TiO}_{2}$ 导带上. 染料结构上另一个重大的设 计就是两个 meso 上的芳基的邻位带有长链烷氧基, 目 的就是抑制卟啉自身 $\pi-\pi$ 堆积, 并且能够有效地抑制电 解质氧化还原电对从 $\mathrm{TiO}_{2}$ 导带上得到电子重组, 从而 抑制暗电流的产生.

近两年卟啉敏化染料光电转换效率提升很大, 但是 卟啉染料在 $450 \sim 550 \mathrm{~nm}$ 之间吸收窄的问题将是制约卟 啉染料效率进一步提升的瓶颈, 国际上很多研究组开始 了新的尝试，一个思路就是通过电子给体的吸收经 $\pi$ 


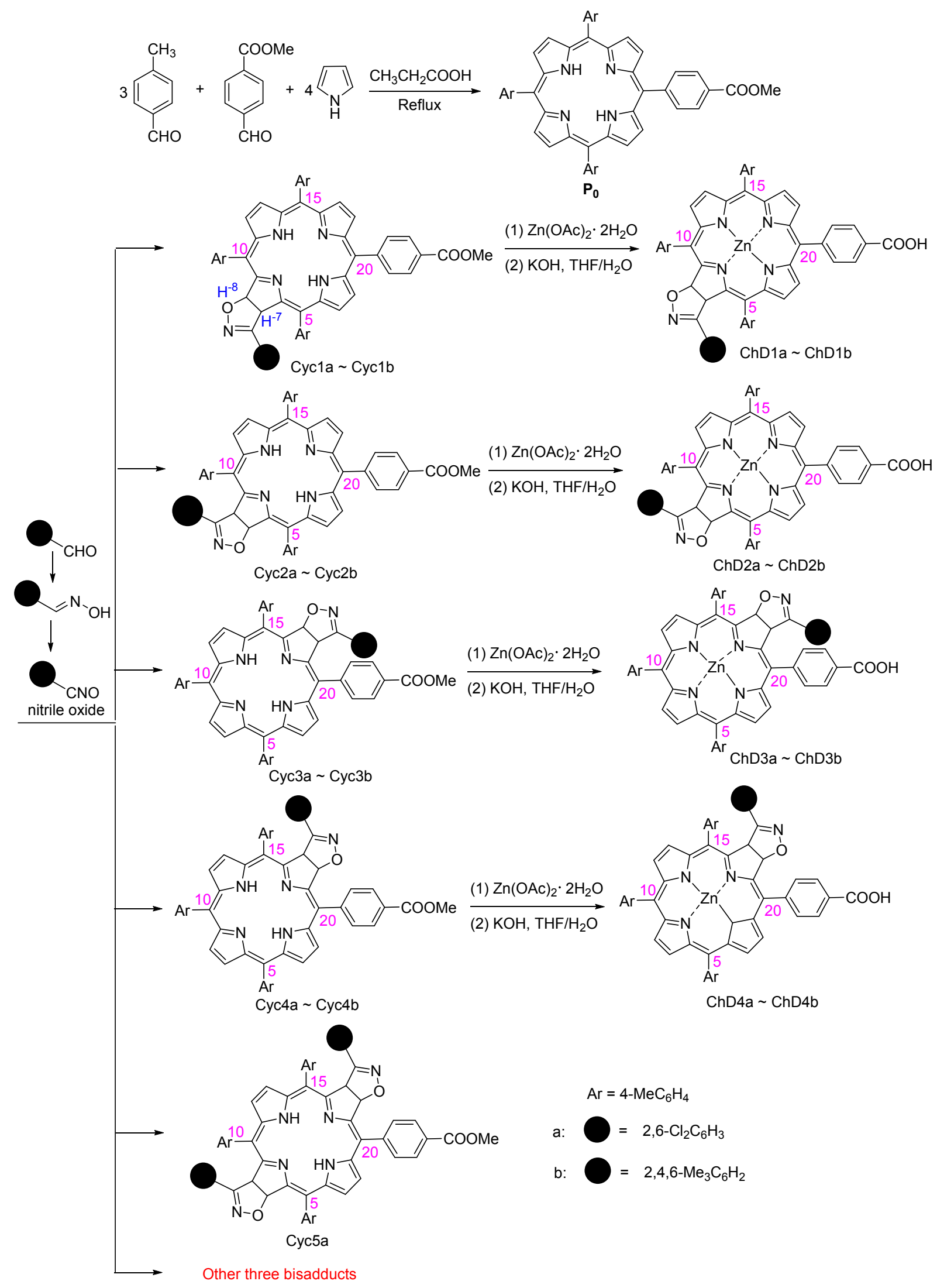

图 6 Feng 设计的 Chlorin 卟啉染料结构

Figure 6 The Chlorin porphyrin dye by Feng group 


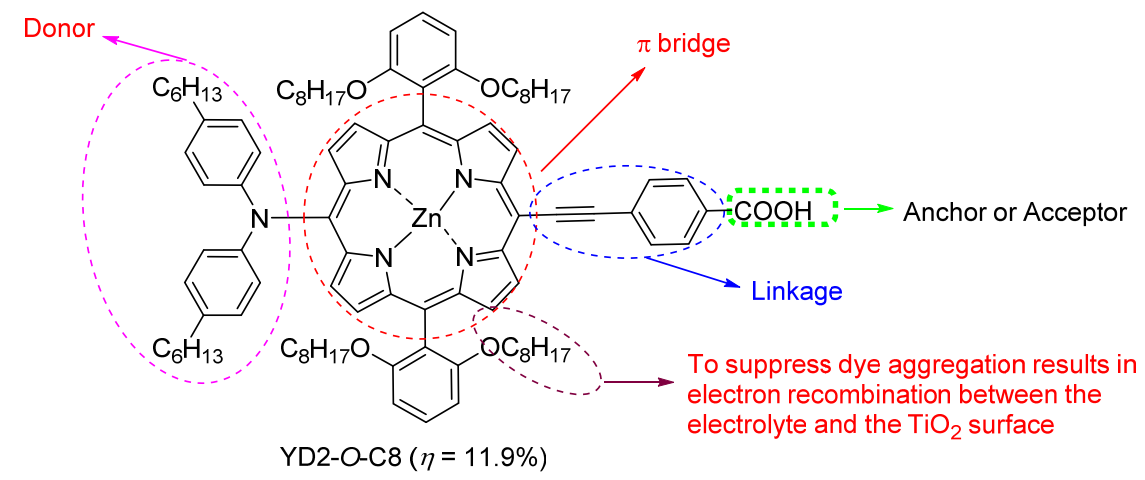

图 $7 \mathrm{YD} 2-\mathrm{O}-\mathrm{C} 8$ 的结构

Figure 7 Structure of YD2-O-C8

电子延伸而使整个染料的吸收红移.

其中王鹏等 ${ }^{[46]}$ 在 YD2- $O-\mathrm{C} 8$ 结构的基础上, 把另一 个强电子给体 $\mathrm{N}$-稠芢基团通过乙炔基连接在卟啉的中 位上, 结构如图 8 的 WW-6, 在同样的测试条件下, WW-6 与 YD2-O-C8 的光电转换效率一样, 达到了
$10.5 \%$.

Diau 及其合作者 ${ }^{[47]}$ 在 YD2-O-C8 结构基础上也设 计了可见光全吸收染料 LD-31(图 9).

通过引入葱环进一步使电子给体的 $\pi$ 电子延伸, 其 最大吸收边可达 $800 \mathrm{~nm}$, 与有机染料 AN-4 共敏化, 获

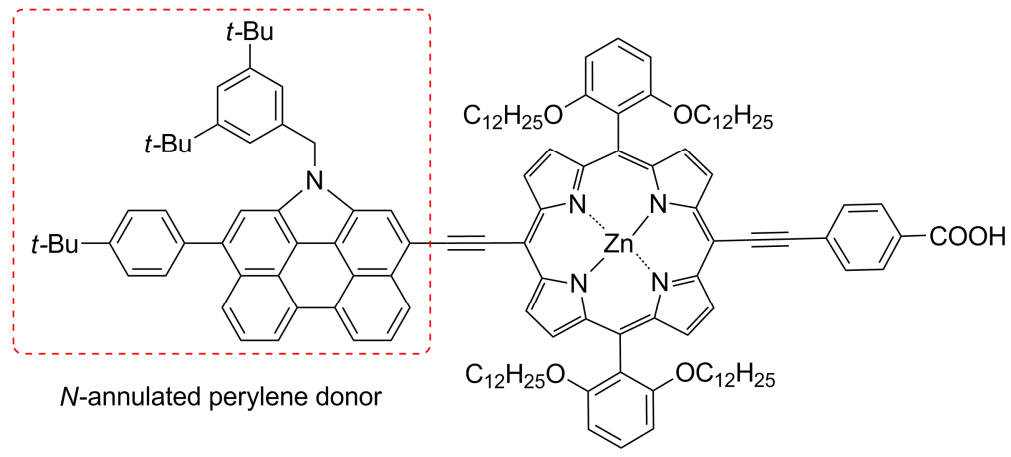

WW-6 ( $\eta=10.5 \%$, reference dye: YD2-O-C8 $\eta=10.5 \%)$

图 8 WW-6 的结构

Figure 8 Structure of WW-6

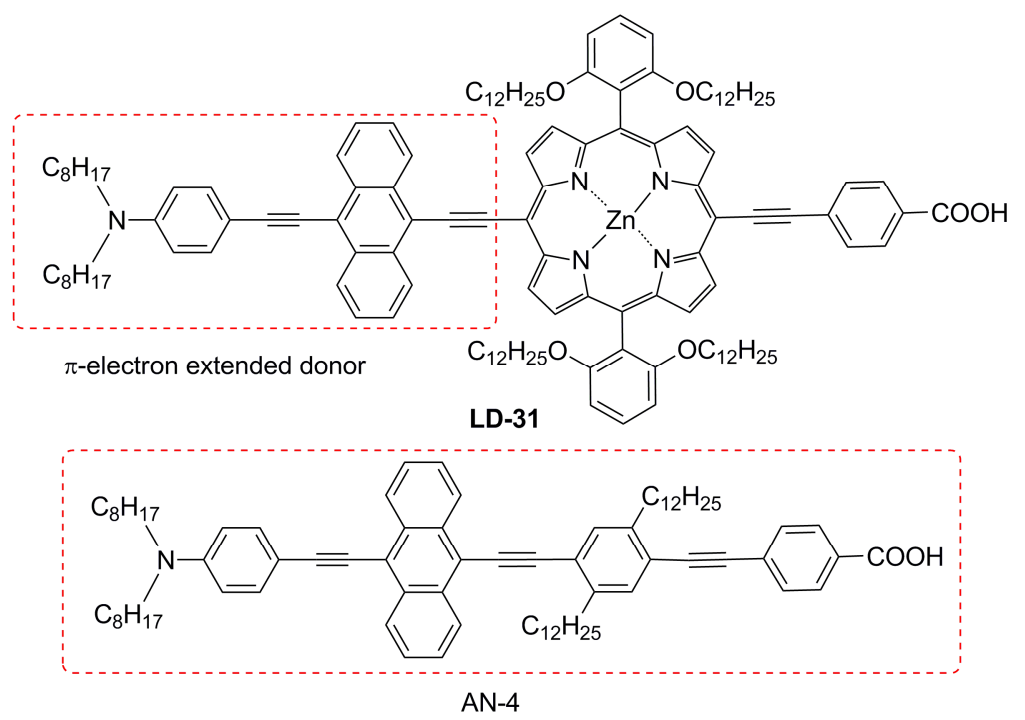

图 9 LD-31 的结构

Figure 9 Structure of LD-31 
得了 $J_{\mathrm{sc}}=20.3 \mathrm{~mA} \cdot \mathrm{cm}^{-2}, V_{\mathrm{oc}}=704 \mathrm{mV}, F F=0.72$, 其 $\eta$ 值达到 $10.3 \%$.

Yeh 和 Grätzel 等 ${ }^{[48]}$ 在 YD2-O-C8 结构的基础上，把 苯并噻二唑插入乙炔桥和苯环之间, 增强拉电子效果, 更有利于电子传递, 整个染料也有一定的红移, 结构如 图 10 的 GY50, 其光电转换效率达到了 $12.75 \%$. 实验还 证实, 苯环作为空间间隔很关键, 如果去掉苯环, 电子 受体直接连在苯并噻二唑上, 光电转换效率下降 6 倍.

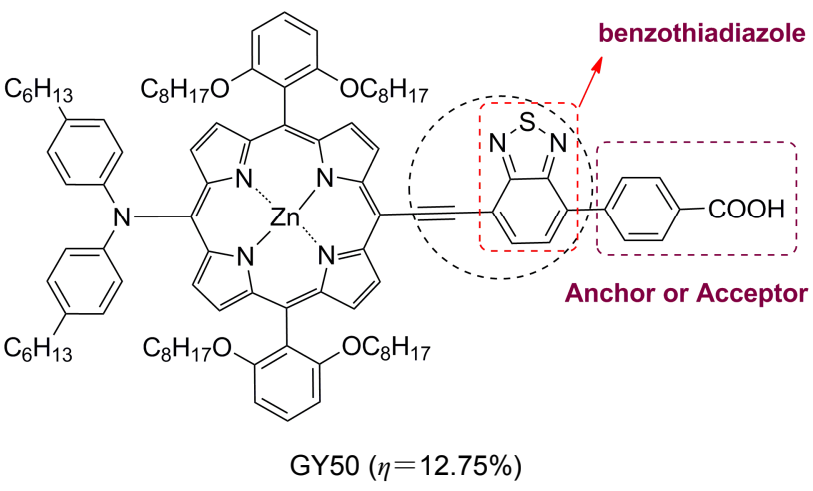

图 10 GY50 的结构

Figure 10 Structure of GY50

Grätzel 等 ${ }^{[49]}$ 继续在 GY50 的基础上, 引入大给体基 才, 加大对位电子给体的 $\pi$ 电子共轭能力, 结构如图 11 的 SM315, 更关键的是在电子给体上又引入了 4 个长链 烷氧基, 总共 8 个长链烷氧基从而更好地抑制钴电解质 相对严重的电荷复合, 其光电转换效率达到了 $13 \%$, 成 为当前效率最高的吓啉染料. 与染料 GY50 的电池性能 参数比较, 染料 SM315 的开路电压大, 而短路电流密度 反而小于 GY50, 但最终的光电转换效率更高, 说明大 给体没有明显提高给电子能力, 反而是多个长链烷烃的
引入更好地抑制了电解质与光生电子的重组, 极大地降 低了暗电流的产生，从而导致更高的光电转换效率. 这 成为今后卟啉染料设计的一个重要思路.

解永树研究组 ${ }^{[50 \mathrm{a} \sim 50 \mathrm{f}]}$ 在卟啉类染料敏化太阳能电池 领域开展了系统的研究, 取得了一系列突出的研究成 果. 该研究组将咔唑和二苯胺噻吩作为给体引入到了卟 啉上，研究了不同给体对染料效率的影响. 他们还在靠 近咔唑给体位置引入额外的炔键来扩展卟啉染料的 $\pi$ 共轭结构，以增强其在近红外区吸收，并同时引入多达 六个烷氧基链来抑制分子间聚集作用，由此合成的卟啉 染料 XW4(图 12)的光电转换效率达到 7.94\%, 与 C1 共 敏化之后，其 $500 \mathrm{~nm}$ 左右的吸收缺陷可以得到很好的 弥补，光电转换效率可进一步明显提高至 $10.45 \%$. 值 得强调的是，他们还进一步设计、合成了几个新颖的共 敏化剂, 可以同时弥补卟啉染料在 $400 \mathrm{~nm}$ 和 $500 \mathrm{~nm}$ 左 右的两个吸收缺陷，有效地提高了电池的短路电流和开 路电压. 最终, 共敏化剂 XS3(图 12)与卟啉染料 XW4 共 敏化得到了高达 $10.75 \%$ 的光电转换效率. 这些研究工 作为高效卟啉染料和共敏化剂的设计、合成提供了重要 的设计策略和思路.

\section{3 结论与展望}

经过近二十年的发展, 卟啉敏化太阳能电池已经取 得了长足的进展，目前在实验室中的光电转换效率已经 超过 $13 \%$. 人们已基本形成共识, 高性能的染料分子必 须满足以下的原则: (1)在可见光谱区甚至在近红外有好 的光捕集能力; (2)有效的光电能量转换, 好的电荷分离; (3)最小化的电子重组, 分离电子和空穴能有效地注入 到相应的电极上. 此外, 这类电池如果要实现实用化,

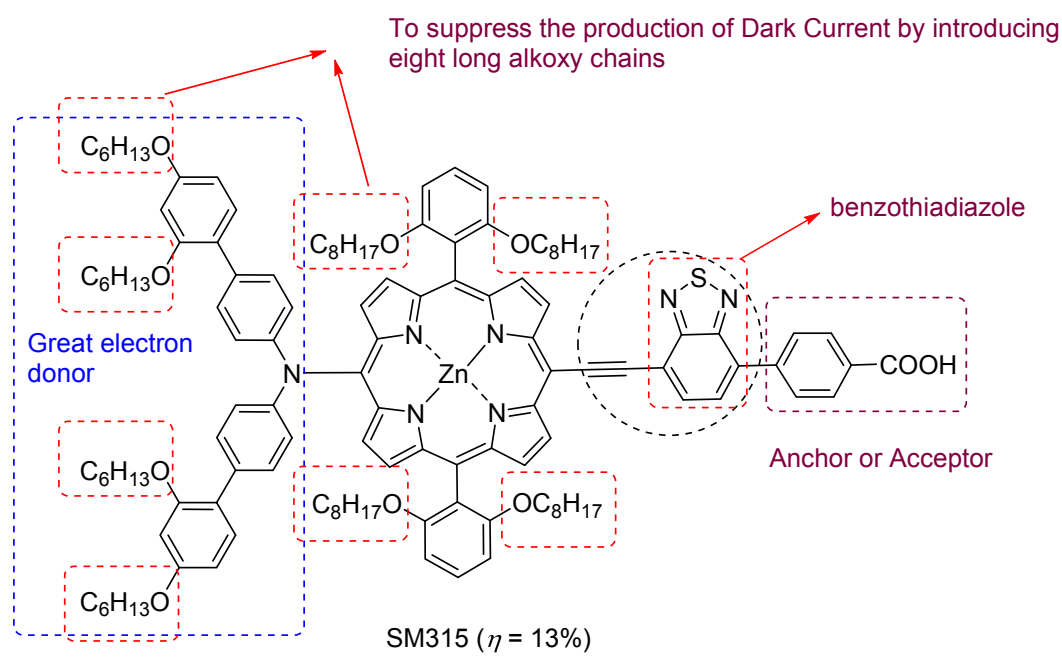

图 $11 \mathrm{SM} 315$ 的结构

Figure 11 Structure of SM315 


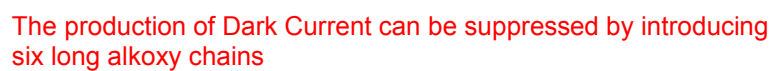
six long alkoxy chains
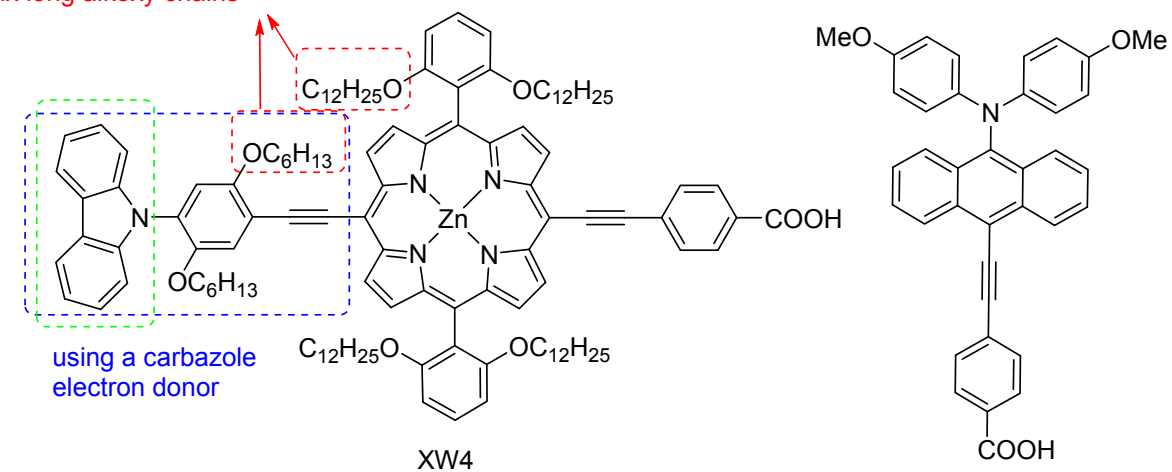

$\mathrm{C} 1$

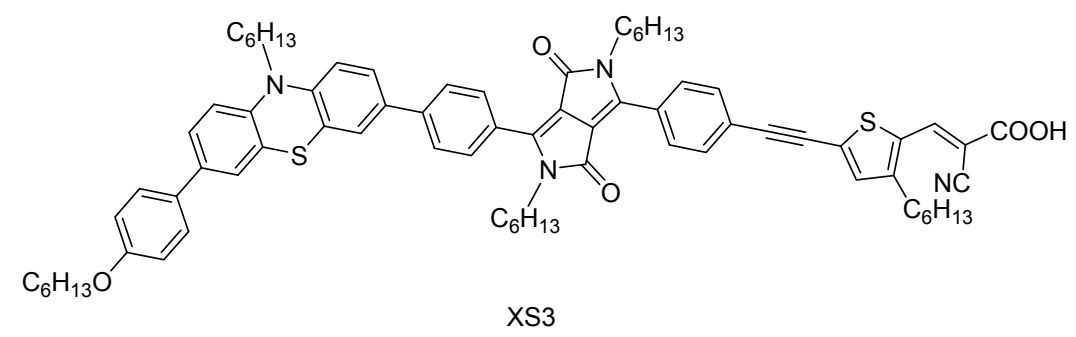

图 12 卟啉染料 XW4 和共敏化剂 C1、XS3 的化学结构

Figure 12 Chemical structures of the porphyrin dye XW4 and the cosensitizers C1 and XS3

还需要解决, 比如大规模制备高效率卟啉染料, 䇻选稳 定性好, 环境友好的电解质, 大面积电池的开发以及光 阳极的优化等.

从染料敏化太阳能电池的工作原理中知道, 光敏染 料是一个非常重要的单元, 染料的性能直接影响到 DSSC 的光电转化效率. 经过多年的发展, 锌卟啉染料 具有价廉、环境友好以及非常高的化学稳定性和突出的 氧化还原特性, 而成为现在最具吸引力一类染料敏化 剂. 高性能锌卟啉染料的研究主要集中在从卟啉 meso 位出发 “推-拉” 电子的 D- $\pi$-A 设计体系上. 然而卟啉 分子尺寸改变不大的情况下, 卟啉染料先天在 $450 \sim 550$ $\mathrm{nm}$ 之间缺少吸收, 离全光谱吸收染料还有一定差距, 目前的主要思路就是通过与一个在此吸收波长范围内 染料共敏化或者加入能量传递染料.

能否实现单一的染料有全光谱响应? 如何克服这 块卟啉先天缺陷是具有重要研究价值的; 此外在已有成 功的高效卟啉染料模型分子的基础上, 企图通过电子给 体增加扩展 $\pi$ 电子从而使吸收相应变宽, 但事实上其效 率反而并没有明显提升, 这说明染料分子尺寸与在纳米 晶 $\mathrm{TiO}_{2}$ 多孔膜上的堆积密度有很大关系. Andersson 等 ${ }^{[51]}$ 在研究 N719 和 Z907 在 $\mathrm{TiO}_{2}$ 纳米膜表面覆盖率与 光电性能时发现, 短路电流密度和光电转换效率随着染 料覆盖率的增加而成线性增加, 而开路电压增加不明 显. 很明显整个染料分子尺寸的加大造成在 $\mathrm{TiO}_{2}$ 薄膜 表面有效堆积密度减少, 从而减少注入 $\mathrm{TiO}_{2}$ 导带电子
的数目. 研究分子尺寸大小与在 $\mathrm{TiO}_{2}$ 薄膜表面有效堆 积密度之间的关系也是很有意义的工作.

综上所述，相比较具有近红外吸收的单晶硅材料, 从分子工程的角度出发，设计分子结构，增强可见光全 吸收, 甚至具有近红外吸收的卟啉染料仍然富有挑战 性，正因为如此，卟啉染料发展潜力巨大.

\section{References}

[1] Dresselhaus, M. S.; Thomas, I. L. Nature 2001, 414, 332.

[2] Imahori, H. J. Mater. Chem. 2007, 17, 31.

[3] Goncalves, L. M.; Bermudez, V. Z.; Ribeiro, H. A.; Mendes, A. M. Energy Environ. Sci. 2008, 1, 655.

[4] Nazeeruddin, M. K.; Péchy, P.; Grätzel, M. Chem. Commun. 1997, $18,1705$.

[5] O'Regan, B.; Grätzel, M. Nature 1991, 353, 737.

[6] Nazeeruddin, M. K.; Kay, A.; Rodicio, I.; Humpbry-Baker, R.; Miiller, E.; Liska, P.; Vlachopoulos, N.; Grätzel, M. J. Am. Chem. Soc. 1993, 115, 6382.

[7] Nazeeruddin, M. K.; Péchy, P.; Renouard, T.; Zakeerddin, S. M.; Humphry-Baker, R.; Comte, P.; Liska, P.; Cevey, L.; Costa, E.; Shklover, V.; Spiccia, L.; Deacon, G. B.; Bignozzi, C. A.; Grätzel, M. J. Am. Chem. Soc. 2001, 123, 1613.

[8] Wang, P.; Zakeeruddin, S. M.; Exnar, I.; Grätzel, M. Chem. Commun. 2002, 2972.

[9] Wang, P.; Zakeeruddin, S. M.; Humphry-Baker, R.; Moser, J. E.; Grätzel, M. Adv. Mater. 2003, 15, 2101.

[10] Wang, P.; Zakeeruddin, S. M.; Moser, J. E.; Nazeeruddin, M. K.; Sekiguchi, T.; Grätzel, M. Nature Mater. 2003, 2, 402.

[11] Grätzel, M. J. Photochem. Photobiol. A 2004, 164, 3.

[12] Nazeeruddin, M. K.; Angelis, F. D.; Fantacci, S.; Selloni, A.; Vis- 
cardi, G.; Liska, P.; Ito, S.; Takeru, B.; Grätzel, M. J. Am. Chem. Soc. 2005, 127, 16835.

[13] Gao, F. F.; Wang, Y.; Shi, D.; Zhang, J.; Wang, M. K.; Jing, X. Y.; Humphry-Baker, R.; Wang, P.; Zakeeruddin, S. M.; Grätzel, M. J. Am. Chem. Soc. 2008, 130, 10720.

[14] Yu, Q.; Wang, Y. H.; Yi, Z. H.; Zu, N. N.; Zhang, J.; Zhang, M.; Wang, P. ACS NANO 2010, 4, 6032.

[15] Peter, L. M. J. Phys. Chem. Lett. 2011, 2, 1861.

[16] (a) Mishra, A.; Fischer, M. K. R.; Bäuerle, P. B. Angew. Chem., Int. Ed. 2009, 48, 2474.

(b) He, J. J.; Chen, S. X.; Wang, T. T.; Zeng, H. P. Chin. J. Org. Chem. 2012, 32, 472 (in Chinese).

(何俊杰, 陈舒欣, 王婷婷, 曾和平, 有机化学, 2012, 32, 472.)

[17] (a) Lindsey, J. S. Acc. Chem. Res. 2010, 43, 300.

(b) Guo, C. C. Chin. J. Org. Chem. 1991, 11, 416 (in Chinese).

(郭灿城, 有机化学, 1991, 11, 416.)

(c) Pan, J. G.; He, M. W. Chin. J. Org. Chem. 1993, 13, 533 (in Chinese).

(潘继刚, 何明威, 有机化学, 1993, 13, 533.)

[18] (a) Wu, D.; Shen, Z.; Xue, Z. L.; You, X. Z. Chin. J. Inorg. Chem. 2007, 23, 1 (in Chinese).

(吴迪, 沈珍, 薛兆历, 游效曾, 无机化学学报, 2007, 23, 1.)

(b) Li, Z.; Jia, C. Y.; Wan, Z. Q. Prog. Chem. 2011, 23, 1014 (in Chinese).

(李孜, 贾春阳, 万中全, 化学进展, 2011, 23, 1014.)

(c) Tang, Y. Y.; Mei, Q. B.; Xu, Z. J.; Lin, Q. D. Prog. Chem. 2011, 23, 1915 (in Chinese).

(汤雅芸, 梅群波, 徐志杰, 凌启淡, 化学进展, 2011, 23, 1915.)

[19] Imahori, H. Key Eng. Mater. 2011, 451, 29.

[20] (a) Li, L. L.; Diau, E. W. Chem. Soc. Rev. 2013, 42, 291.

(b) Urbani, M.; Grätzel, M.; Nazeeruddin, M. K.; Torres, T. Chem. Rev. 2014, 114, 12330.

[21] Kay, A.; Grätzel, M. J. Phys. Chem. 1993, 97, 6272.

[22] Nazeeruddin, M. K.; Humphry-Baker, R.; Officer, D. L.; Campbell, W. M.; Burrell, A. K.; Grätzel, M. Langmuir 2004, $20,6514$.

[23] Wang, Q.; Campbell, W. M.; Bonfantani, E. E.; Jolley, K. W.; Officer, D. L.; Walsh, P. J.; Gordon, K.; Humphry-Baker, R.; Nazeeruddin, M. K.; Grätzel, M. J. Phys. Chem. B 2005, 109, 15397.

[24] Campbell, W. M.; Jolley, K. W.; Wagner, P.; Wagner, K.; Walsh, P. J.; Gordon, K. C.; Mende, L. S.; Nazeeruddin, M. K.; Wang, Q.; Grätzel, M.; Officer, D. L. J. Phys. Chem. C 2007, 111, 11760.

[25] Mishra, A.; Fischer, M. K. R.; Bäuerle, P. Angew. Chem., Int. Ed. 2009, 48, 2474.

[26] Lin, C. Y.; Lo, C. F.; Luo, L. Y.; Lu, H. P.; Hung, C. S.; Diau, E. W. G. J. Phys. Chem. C 2009, 113, 755.

[27] Liu, Y. J.; Xiang, N.; Feng, X. M.; Shen, P.; Zhou, W. P.; Weng, C.; Zhao, B.; Tan, S. T. Chem. Commun. 2009, 18, 2499.

[28] Ma, R.; Guo, P.; Cui, H. J.; Zhang, X. X.; Nazeeruddin, M. K.; Grätzel, M. J. Phys. Chem. A 2009, 113, 10119.

[29] Tanaka, M.; Hayashi, S.; Eu, S.; Umeyama, T.; Matano, Y.; Imahori, H. Chem. Commun. 2007, 2069.

[30] Hayashi, S.; Tanaka, M.; Hayashi, H.; Eu, S.; Umeyama, T.; Matano, Y.; Araki, Y.; Imahori, H. J. Phys. Chem. C 2008, 112, 15576.

[31] Hayashi, S.; Matsubara, Y.; Eu, S.; Hayashi, H.; Umeyama, T.; Matano, Y.; Imahori, H. Chem. Lett. 2008, 37, 846.
[32] Eu, S.; Hayashi, S.; Umeyama, T.; Matano, Y.; Araki, Y.; Imahori, H. J. Phys. Chem. C 2008, 112, 4396.

[33] Park, J. K.; Chen, J. P.; Lee, H. R.; Park, S. W.; Shinokubo, H.; Osuka, A.; Kim, D. J. Phys. Chem. C 2009, 113, 21956.

[34] Mozer, A. J.; Griffith, M. J.; Tsekouras, G.; Wagner, P.; Wallace, G. G.; Mori, S.; Sunahara, K.; Miyashita, M.; Earles, J. C.; Gordon, K. C.; Du, L.; Katoh, R.; Furube, A.; Officer, D. L. J. Am. Chem. Soc. 2009, 131, 15621.

[35] (a) Liu, P. P.; Feng, Y. Q.; Gu, C. Z.; Meng, S. X.; Zhang, B. Chin. Chem. Lett. 2012, 23(5), 505.

(b) Yan, J. Y.; Feng, Y. Q.; Peng, X.; Li, Y. C.; Zhang, N. N.; Li, X. G.; Zhang, B. Tetrahedron Lett. 2013, 54, 7198.

(c) Zhang, N. N.; Feng, Y. Q.; Li, Y. C.; Peng, X.; Gu, C. Z.; Xue, X. D.; Yan, J. Y.; Chen, Q. L.; Li, X. G.; Zhang, B. New J. Chem. 2013, 37, 1134.

(d) Xue, X. D.; Zhang, W. H.; Feng, Y. Q.; Zhang, N. N.; Ju, C. G.; Peng, X.; Yang, Y. B.; Zhang, B. RSC Adv. 2014, 4, 8894.

(e) Zeng, Z.; Zhang, B.; Li, C. J.; Peng, X.; Liu, X. J.; Meng, S. X.; Feng, Y. Q. Dyes Pigm. 2014, 100, 278,

[36] Liu, X. J.; Li, C. J.; Peng, X.; Zhou, Y. Z.; Zeng, Z.; Li, Y. C.; Zhang, T. Y.; Zhang, B.; Dong, Y.; Sun, D. M.; Cheng, P.; Feng, Y. Q. Dyes Pigm. 2013, 98, 181.

[37] Lee, C. W.; Lu, H. P.; Lan, C. M.; Huag, Y. L.; Liang, Y. R.; Yen, W. N.; Liu, Y. C.; Lin, Y. S.; Diau, E. W. G.; Yeh, C. Y. Chem. Eur. J. 2009, 15, 1403.

[38] Lin, C. Y.; Lo, C. F.; Luo, L. Y.; Lu, H. P.; Hung, C. S.; Diau, E. W. G. J. Phys. Chem. C 2009, 113, 755.

[39] Lu, H. P.; Mai, C. L.; Tsia, C. Y.; Hsu, S. J.; Hsieh, C. P.; Chiu, C. L.; Yeh, C. Y.; Diau, E. W. G. Phys. Chem. Chem. Phys. 2009, 11, 10270 .

[40] Lin, C. Y.; Wang, Y. C.; Hsu, S. J.; Lo, C. F.; Diau, E. W. G. J. Phys. Chem. C 2010, 114, 687.

[41] Mai, C. L.; Huang, W. K.; Lu, H. P.; Lee, C. W.; Chiu, C. L.; Liang, Y. R.; Diau, E. W. G.; Yeh, C. Y. Chem. Commun. 2010, 46, 809.

[42] Hsieh, C. P.; Lu, H. P.; Chiu, C. L.; Lee, C. W.; Chung, S. H.; Mai, C. L.; Yen, W. N.; Hsu, S. J.; Diau, E. W. G.; Yeh, C. Y. J. Mater. Chem. 2010, 20, 1127.

[43] Wu, S. L.; Lu, H. P.; Yu, H. T.; Chuang, S. H.; Chiu, C. L.; Lee, C. W.; Diau, E. W. G.; Yeh, C. Y. Energy Environ. Sci., 2010, 3, 949.

[44] Sanchis, T. R.; Guo, B. C.; Wu, H. P.; Pan, T. Y.; Lee, H. W.; Raga, S.; Santiago, F. F.; Bisquert, J.; Yeh, C. Y.; Diau, E. W. G. Chem. Commun. 2012, 48, 4368.

[45] Yella, A.; Lee, H. W.; Tsao, H. N.; Yi, C. Y.; Chandiran, A. K.; Nazeeruddin, M. K.; Diau, E. W. G.; Yeh, C. Y.; Zakeeruddin, S. M.; Grätzel, M. Science 2011, 334, 629.

[46] Luo, J.; Xu, M. F.; Li, R. Z.; Huang, K. W.; Jiang, C. Y.; Qi, Q. B.; Zeng, W. D.; Zhang, J.; Chi, C. Y.; Wang, P.; Wu, J. S. J. Am. Chem. Soc. 2014, 136, 265

[47] Wang, C. L.; Hu, J. Y.; Wu, C. H.; Kuo, H. H.; Chang, Y. C.; Lan, Z. J.; Wu, H. P.; Diau E. W. G.; Lin. C. Y. Energy Environ. Sci. 2014, 7, 1392.

[48] Yella, A.; Mai, C. L.; Zakeeruddin, S. M.; Chang, S. N.; Hsieh, C. H.; Yeh, C. Y.; Grätzel, M. Angew. Chem., Int. Ed. 2014, 53, 2973.

[49] Mathew, S.; Yella, A.; Gao, P.; Humphry-Baker, R.; Curchod, B. F. E.; Ashari-Astani, N.; Tavernelli, I.; Rothlisberger, U.; Nazeeruddin, M. K.; Grätzel, M. Nature Chem. 2014, 6, 242. 
[50] (a) Liu, B.; Zhu, W. H.; Wang, Y. Q.; Wu, W. J.; Li, X.; Chen, B. Q.; Long, Y. T.; Xie, Y. S. J. Mater. Chem. 2012, 22, 7434.

(b) Chen, B.; Li, X.; Wu, W. J.; Zha, Q. Z.; Xie, Y. S. RSC Adv. 2014, 4, 10439

(c) Wang, Y. Q.; Li, X.; Liu, B.; Wu, W. J.; Zhu, W. H.; Xie, Y. S. RSC Adv. 2013, 3, 14780.

(d) Wang, Y. Q.; Xu, L.; Wei, X. D.; Li, X.; Ågren, H.; Wu, W. J.;
Xie, Y. S. New J. Chem. 2014, 38, 3227.

(e) Wang, Y. Q.; Chen, B.; Wu, W. J.; Li, X.; Zhu, W. H.; Tian, H.; Xie, Y. S. Angew. Chem., Int. Ed. 2014, 53, 10779.

(f) Sun, X.; Wang, Y. Q.; Li, X.; Ågren, H.; Zhu, W. H.; Tian, H.; Xie, Y. S. Chem. Commun. 2014, 50, 15609.

[51] Johansson, V.; Gibbings, L. E.; Clarke, T.; Gorlov, M.; Andersson, G. G.; Kloo, L. Phys. Chem. Chem. Phys. 2014, 16, 711.

(Cheng, F.) 